\title{
Common origin of doping-limiting mechanisms in IIB-VI compounds and alloys
}

\author{
Uroš V. Desnica*, Ida Dunja Desnica-Frankovic \\ Semiconductors Laboratory, Division of Materials Physics, Physics Department, R. Boškovic Institute, Bijenicka 54, \\ 10000 Zagreb, Croatia
}

\begin{abstract}
Wide-band-gap II-VI semiconductors have a potential for a variety of applications, which are presently hindered by the difficulties in achieving efficient doping at both $\mathrm{n}$ - and p-side (above $10^{18} \mathrm{~cm}^{-3}$ ). The origin and even more the microscopic nature of doping problems, remain controversial. In this paper, we have shown that for IIB-VI compounds or alloys the common origin of very different doping-limiting mechanisms can be traced down to the ratio of covalent radii of the constituent atoms (and respective vacancies). This ratio then determines the ratio of vacancy formation energies, and finally the ratio of their relative concentrations. The practical consequence is that the $\mathrm{n}$ - and $\mathrm{p}$-type dopability of IIB-VI compounds as well as their ternary alloys can be predicted in a simple way. Limits of the approach are discussed. (C) 2001 Elsevier Science Ltd. All rights reserved.
\end{abstract}

Keywords: Doping; II-VI; P-type; N-type; Compensation; A centre; DX centre

\section{Introduction}

Wide-band-gap II-VI compounds are technologically very interesting semiconductors, but for all applications it is essential to achieve a good bipolar electrical conductivity, i.e. to accomplish efficient doping both with donors and acceptors up to very high concentrations (above $10^{18} \mathrm{~cm}^{-3}$, and desirably above $10^{19} \mathrm{~cm}^{-3}$ ). However, difficulties in accomplishing efficient doping of the wide-band-gap II-VI compounds of both p- and/or n-type still remain main obstacles in applications but also

\footnotetext{
*Corresponding author. Tel.: + 3851-4561-173; fax: + 3851-4680-114.

E-mail address: desnica@rudjer.irb.hr(U.V. Desnica).
}

a continuous puzzle about this whole class of materials which has not yet been fully solved.

The first blue laser based on ZnSe in 1991 has revived the interest for II-VI compounds and inspired numerous theoretical and experimental studies of the mechanisms that are responsible for doping difficulties. Specifically, these problems are claimed to be self-compensatory by spontaneously generated oppositely charged native defects $[1,2]$, which can make compensating pairs with dopants (A centres) $[3,4]$, strong lattice relaxation around the doping atom resulting in the formation of compensating deep localised levels [5,6], mid-gap pinning of the Fermi level $[7,8]$, amphoteric behaviour of some dopants [9], or insufficient solubility of others [9].

In this paper we demonstrate that the relative size of vacancies in the IIB or VI sublattice is 
a common underlying factor, irrespective of the mechanism which actually limits doping in a particular dopant/compound combination.

\section{The role of native vacancies as possible compensating species}

Historically, in IIB-VI compounds, spontaneously created native vacancies either in the IIB sublattice (which are acceptors) or in the group-VI sublattice (which are donors) have been the first [1], and long lasting suspects [10] as compensating defects. However, the analysis of all experimental and newer theoretical vacancy-related results suggest that isolated native vacancies are not as important as assumed earlier.

Better quantitative experimental determination of the concentration of isolated native vacancies showed that very few of them survive cooling from high to room temperature. It is now evident that, in undoped samples, the residual impurities control the electrical properties, not the vacancies. In doped IIB-VIs, larger vacancy concentrations were observed only if they pair with dopant atoms (A centres) (for references see review papers [11] or [12]). Additional arguments came from newer and more precise calculations (when the formation energies of all native defects were predicted by $\mathrm{ab}$ initio calculations $[9,13]$ ) which show that the concentrations of all native defects, vacancies included, are too low to compensate dopants significantly.

Hence, a consensus has been reached [3,11-15], based both on experimental and theoretical results, that isolated vacancies are not a direct cause of dopant deactivation and that isolated vacancies are never dominant defects.

\section{Indirect influence of vacancies on the doping efficiency}

Since the concentration of isolated vacancies is generally low and their formation energies considerably higher than those of many dopants, they can be excluded as compensating species that directly deactivate dopants in IIB-VIs. However, in this section, we will shows that isolated vacancies, in an indirect way, still play an extremely important, and in fact decisive role in doping efficiency.

\subsection{Theoretical considerations}

The equilibrium concentration of each dopant or defect, $\left[N_{j}\right]$, is defined by its formation energy, and can be expressed as [9]

$\left[N_{j}\right]=\left[N_{\text {sites }}\right] \exp \left(-E_{\text {form }}\left(N_{j}\right) / k T\right)$,

where $E_{\mathrm{form}}\left(N_{j}\right)$ is the formation energy of the $j$ th defect or dopant, whereas $\left[N_{\text {sites }}\right]$ is the concentration of possible defect sites. Applying Eq. (1) to both types of vacancies, $V_{\mathrm{II}}$ and $V_{\mathrm{VI}}$, and taking into account that $\left[N_{\text {sites }}\right]$ is the same for both sublattices, one obtains

$k T \ln \left(\left[V_{\mathrm{II}}\right] /\left[V_{\mathrm{VI}}\right]\right)=E_{\text {form }}\left(V_{\mathrm{VI}}\right) / E_{\text {form }}\left(V_{\mathrm{II}}\right)$.

The vacancy formation energy depends directly on its size [16], simply because more energy is needed to form larger cavity in a crystal. On the other hand, the size of the vacancy corresponds to the size of the respective atom, and can be described with its covalent radius, $r_{\mathrm{c}}(\mathrm{II})$, or $r_{\mathrm{c}}(\mathrm{VI})$. Hence, the ratio of covalent radii, $R=r_{\mathrm{c}}(\mathrm{II}) / r_{\mathrm{c}}(\mathrm{VI})$ determines the ratio of the formation energies for vacancies at both lattice sites, and, according to Eq. (2), the ratio of respective vacancy concentrations. Since $V_{V I}$ are donors and $V_{\mathrm{II}}$ are acceptors, large $R$ means more native donors than acceptors, and vice versa. This calculated result coincides with natural preferences in all undoped IIB-VIs: all compounds with large $R$ are n-type and vice versa, while CdTe, being the only II-VI that can be doped both types, has very similar $r_{\mathrm{c}}(\mathrm{II})$ and $r_{\mathrm{c}}(\mathrm{VI})$. It seems unlikely that this coincidence is purely accidental, so the ratio $R$ (reflecting the ratio $\left[V_{\mathrm{VI}}\right] /\left[V_{\mathrm{II}}\right]$ ) might be an important factor for the success of dopability in IIB-VIs, despite the fact that both $\left[V_{\mathrm{VI}}\right]$ and $\left[V_{\mathrm{II}}\right]$ are small in comparison with dopant concentrations and thus irrelevant as compensating species, as discussed in Section 2.

\subsection{Experimental considerations}

Figs. 1 and 2 summarize the compilation of best experimental doping results in IIB-VI compounds 


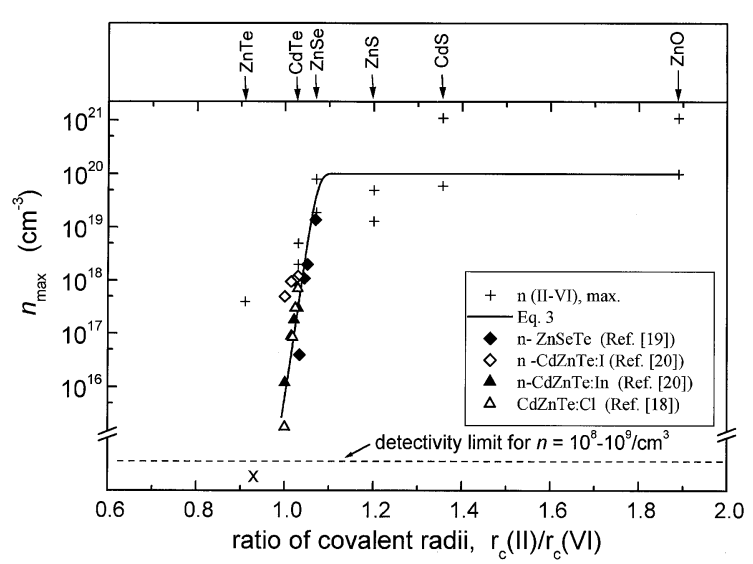

Fig. 1. Maximal free electron concentrations, $n_{\max }$, achieved in IIB-VI compounds or alloys for growth/doping under the equilibrium or non-equilibrium conditions. References for alloys are indicated in the figure, while most of the values for binary compounds were taken from Refs. [12] and [23]. Higher value refers to non-equilibrium, and lower to the equilibrium conditions. Symbol $\times$ indicates cases where $n$-doping was impossible to achieve with equilibrium approaches.

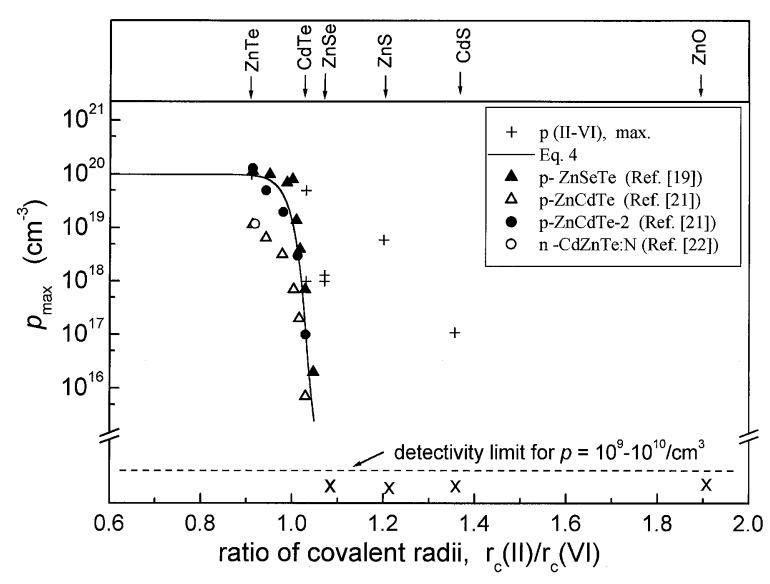

Fig. 2. Maximal free hole concentrations, $p_{\max }$, achieved in IIB-VI compounds or alloys for growth/doping under the equilibrium or non-equilibrium conditions. References for alloys are indicated in the figure, while most of the references for binary compounds were taken from Refs. [12] and [23]. $\times$ indicates cases where p-doping was impossible to achieve with equilibrium approaches.

and ternary IIB-VI alloys [12,18-23], obtained under quasi-equilibrium and non-equilibrium conditions. Cases of traditionally 'difficult' doping, where $\mathrm{p}$ - or n-doping was impossible to achieve for decades, are indicated $(\times)$. In Fig. 1, maximal free electron concentration, $n_{\max }$, is presented as a function of the ratio $R$ of atomic covalent radii of IIB and VI constituents for each IIB-VI compound, in accordance with theoretical arguments given in Section 3.1. Ratios for alloys were calculated using mean values of the radii assuming linear extrapolation. Doping efficiency obviously dramatically depends on $R$, varying from immeasurably low up to excellent doping $\left(10^{20} \mathrm{~cm}^{-3}\right.$ or even higher). The results for maximally obtained n-doping for compounds and alloys under conditions of thermodynamic quasi-equilibrium were fitted to the function:

$n_{\max }=n_{\mathrm{o}, \max }\left[1-\exp \left(-A \times R^{B}\right)\right]$,

where $A$ and $B$ are constants, which are the same for all compounds/alloys, while $n_{\mathrm{o} \text {,max }}$ denotes maximal obtainable concentration.

Fig. 2 refers to acceptors. A similarly simple relation appropriately describes best experimental results obtained for equilibrium conditions:

$p_{\max }=p_{0, \max } \exp \left[-\left(C \times R^{D}\right)\right]$,

where $C$ and $D$ are constants.

In compounds for which two or more values of $n_{\max }$ and $p_{\max }$ are listed, highest values refer to non-equilibrium experimental conditions. Those values again strongly, but less dramatically, depend on $R$ and could be equally well described with analogous simple exponential relations, but with different parameters $A-D$.

Results presented in Figs. 1 and 2 are even more persuasive if one remembers that here collected data were obtained by different growth techniques, different combinations of compound/alloy and dopants, different experimental optimisations, and different authors/labs.

In all these cases, maximally obtained equilibrium carrier concentrations were limited by very different microscopic mechanisms (self-compensation by A centre for CdTe and ZnSe, DX centre for most of the ranges in $\mathrm{ZnCdSe}$ and $\mathrm{CdZnTe}$ alloys and solubility limit for $\mathrm{CdS}$ [12]). The identical dependence on a single variable, $R$, suggests that all 
these mechanisms have a common denominator and possibly a common origin. The existence of a common root is understandable, since all these mechanisms, although apparently very different at the microscopic level, stem from the same basic thermodynamic requirement for minimum energy of the system, which seems to be well described solely by the ratio $R$.

Hence, the vacancy ratio emerges as predominantly responsible for the success of doping, despite the fact that the concentration of either vacancy is never high enough to directly control the conductivity nor to be the major compensating defect. The precise mechanism of how the $\left[V_{\mathrm{II}}\right] /\left[V_{\mathrm{VI}}\right]$ ratio influences dopability is not fully understood as yet. However, its high value obviously facilitates the incorporation and/or electrical activation of donors in very high concentrations without compensation, while at the same time inhibits the incorporation/activation of acceptors. In contrast, a low $\left[V_{\mathrm{II}}\right] /\left[V_{\mathrm{VI}}\right]$ ratio promotes acceptor dopants and strongly hinders donor dopants. Either way, $R$ influences dramatically the possibility of reaching very high levels of both n- and p-type doping in IIB-VI compounds and their alloys.

Understandably, the presented approach has its limits. In particular, it could not be equally well extended to the technologically very interesting and relatively new IIA-VI compounds. A possible explanation could be that, among numerous other differences, in the whole range of lattice constants IIA-VIs have considerably (typically $2 \mathrm{eV}$ ) larger band gaps, $E_{\mathrm{g}}$, than IIB-VIs. Since the tendency of self-compensation dramatically increases with $E_{\mathrm{g}}[1]$, the very large $E_{\mathrm{g}}$ can override the influence of $R$ alone. In such a case the value of $E_{\mathrm{g}}$, as well as the absolute position of $E_{\mathrm{g}}$ with respect to the vacuum level [17], can become the dominant factor which determines maximal dopability.

\section{Conclusion}

Although the concentration of isolated native vacancies in IIB-VIs is relatively low and thus insignificant in governing electrical properties, the relative ease of vacancy formation in one or the other sublattice bears a crucial significance for the efficient doping. The $\left[V_{\mathrm{VI}}\right] /\left[V_{\mathrm{II}}\right]$ ratio is a factor that predominantly influences the success of doping in IIB-VI binary or ternary compounds. It determines a natural tendency of the nominally 'undoped' crystal to have p- or n-type conductivity or both, and it is also a very important factor in either helping or inhibiting the possibility of reaching very high doping levels in IIB-VI compounds and alloys. Since the ratio of concentrations of the respective vacancies depends primarily on the sizes of IIB and VI atoms, which are well known, the $\mathrm{n}$ - and p-type dopability of IIB-VI compounds as well as their ternary alloys can be predicted in a simple way. More importantly, it enables the engineering of ternary alloys by choosing $R$ (i.e. proper fractions of constituents) to be optimised for a sufficiently high doping from both $\mathrm{n}$ - and $\mathrm{p}$-sides.

\section{Acknowledgements}

This research was supported by the Ministry of Science and Technology of Croatia.

\section{References}

[1] Mandel G. Phys Rev 1964;134A:1073.

[2] Marfaing Y. Prog Crystal Growth Charact 1981;4:317.

[3] Marfaing Y. J Crystal Growth 1996;161:205.

[4] Garcia A, Northrup JE. Phys Rev Lett 1995;74:1131.

[5] Chadi DJ. Phys Rev Lett 1994;72:534.

[6] Chadi DJ. Phys Rev B 1999;59:15181.

[7] Hirano K, Suemune I, Sato G. Jpn J Appl Phys 1997;36A/B:L37.

[8] Faschinger W. J Crystal Growth 1996;159:221.

[9] Laks DB, Van de Walle CG, Neumark GF, Bloech PE, Pantelides ST. Phys Rev B 1992;45:10965.

[10] Ichimura M, Wada T, Fujita Sz, Fujita Sg. Jpn J Appl Phys 1991;30:3475.

[11] Neumark GF. Mater Sci Eng 1997;R21:1.

[12] Desnica UV. Prog Crystal Growth Charact 1998;36: 291.

[13] Laks DB, Van de Walle CG. Physica B 1993;185:118.

[14] Prior KA. J Crystal Growth 1995;187:379.

[15] Desnica UV. Vacuum 1998;50:471.

[16] van Vechten JA. Handbook on semiconductors. vol. 3, 1982. p. 1.

[17] Fashinger W. J Crystal Growth 1999;197:557. 
[18] Burkey BC, Khosla RP, Fisher JR, Losee DL. J Appl Phys 1976;47:1095.

[19] Fashinger W, Krump R, Brunhaler G, Ferreira S, Sitter H. Appl Phys Lett 1994;65:3215.

[20] Brun-Le-Cunff D, Baron T, Daudin B, Tatarenko S, Blanchard B. Appl Phys Lett 1995;67:965.
[21] Baron T, Saminadayar K, Magnea N. Appl Phys 1998;83:1354.

[22] Noda D, Aoki T, Nakanishi Y, Hatanaka Y. J Electrochem Soc 1999;146:3482.

[23] Zhang SB, Wei S-H, Zunger A. Appl Phys 1998;83:3192. 